\title{
Efecto del acompañamiento pedagógico en el estrés académico y la ansiedad ante los exámenes, en el contexto covid 19, en estudiantes de una universidad privada Chimbote -2021
}

\author{
Jairo Jaime Turriate Chávez \\ turriatejairo@gmail.com \\ https://orcid.org/0000-0003-3238-251X
}

Carmen Cecilia Torres Ceclén cacetoce1003@hotmail.com https://orcid.org/0000-0002-8616-7965

Lucio Carlos Martos Ramírez carmar2204@hotmail.com https://orcid.org/0000-0002-2732-6452

Universidad César Vallejo RESUMEN

Chimbote - Perú

Introducción: Mena et al. (2021) sostienen que la pandemia ha producido reacciones de todo tipo en la sociedad, particularmente en estudiantes de nivel superior, afectando su estabilidad emocional. Objetivo: Determinar el efecto del acompañamiento pedagógico en el estrés académico y ansiedad ante los exámenes, en el contexto COVID 19, en estudiantes de una universidad privada Chimbote-2021. Esta fue una investigación de tipo pre experimental con una muestra de 136 estudiantes de primer ciclo de pre grado entre los meses marzo a julio de 2021 por medio de un muestreo no probabilístico por conveniencia. Método: cuantitativo y diseño cuasiexperimental, los instrumentos empleados fueron el Cuestionario de Ansiedad ante los exámenes de Valera (CAEX, 1999) y el Inventario de estrés académico de Barraza (SISCO, 2002) seleccionados mediante muestreo de conveniencia y criterios de inclusión. Resultados: como resultado el valor de $Z=-5.766$ y una probabilidad $\mathrm{p}<0.05$, mientras que para ansiedad ante lo exámenes se obtuvo el valor de $\mathrm{Z}=-8.080$ y una probabilidad $\mathrm{p}<0.05$. Conclusiones: Se concluye que el acompañamiento pedagógico tiene un efecto altamente significativo en la disminución del nivel de estrés académico y ansiedad ante los exámenes en el contexto COVID 19, en estudiantes de una universidad privada Chimbote - 2021.

Palabras clave: acompañamiento; estrés; ansiedad. 


\title{
Effect of pedagogical accompaniment on academic stress and anxiety before exams, in the context of COVID 19, in students of a private university Chimbote -2021
}

\begin{abstract}
Introduction: Mena et al. (2021) argue that the pandemic has produced reactions of all kinds in society, particularly in higher level students, affecting their emotional stability. Objective: To determine the effect of pedagogical accompaniment on academic stress and anxiety before exams, in the context of COVID 19, in students of a private university Chimbote-2021. This was a pre-experimental research with a sample of 136 undergraduate students between March and July 2021 through a non-probabilistic sampling for convenience. Method: quantitative and quasi-experimental design, the instruments used were the Anxiety Questionnaire before the Valera exams (CAEX, 1999) and the Barraza Academic Stress Inventory (SISCO, 2002) selected by convenience sampling and inclusion criteria. Results: as a result, the value of $\mathrm{Z}=-5.766$ and a probability $\mathrm{p}<0.05$, while for anxiety before the exams the value of $Z=-8.080$ and a probability $\mathrm{p}<0.05$ were obtained. Conclusions: It is concluded that pedagogical accompaniment has a highly significant effect on the reduction of the level of academic stress and anxiety before the exams in the context of COVID 19, in students of a private university Chimbote - 2021.
\end{abstract}

Keywords: accompaniment; stress; anxiety

Artículo recibido: 15 enero 2022 Aceptado para publicación: 08 febrero 2022 Correspondencia: carmar2204@ hotmail.com Conflictos de Interés: Ninguna que declarar 
Turriate Chávez y otros...

\section{INTRODUCCIÓN}

\subsection{Educación en contexto COVID-19.}

La proliferación del virus SARS-COV 2 (COVID-19) ha desencadenado una crisis en diversos contextos: social, laboral, educativo, entre otros. En el aspecto educativo, se vieron interrumpidas las actividades de tipo presencial a fin de poder mitigar la proliferación del virus. De acuerdo con la Organización de las Naciones Unidas para la educación, ciencia y cultura (UNESCO), 1200 millones de educandos dejaron de asistir presencialmente a su centro de estudios, de los cuales, 160 millones son provenientes de América Latina y el Caribe. Por tanto, los países de la región se vieron obligados a implementar estrategias que les permitan continuar ofertando su servicio educativo para lo cual se valieron de: uso de plataformas digitales, apoyo y desplazamiento de sus colaboradores para que continúen con su labor remotamente, y el bienestar de sus estudiantes íntegramente, según el informe COVID-19 de CEPAL-UNESCO (2020).

Mena et al. (2021) sostienen que la pandemia ha producido reacciones de todo tipo en la sociedad, particularmente en los estudiantes a consecuencia de los cambios que han implementado sus instituciones educativas, sobre todo en el nivel superior. Por tanto, se requiere conocer los niveles de estrés y de qué estrategias se valen estos estudiantes para lidiar el impacto académico y social a raíz de la pandemia. Asimismo, Sánchez et al. (2021) coinciden en sostener las secuelas físicas, psicológicas y conductuales presentadas en este contexto, por ejemplo: depresión y ansiedad por la constante exposición al peligro, así como somatizar y la evitación experiencial sobre todo a inicios de la pandemia como consecuencia del confinamiento y las medidas restrictivas implantadas en sus países.

\subsection{Ansiedad en estudiantes universitarios}

Vivanco et al. (2020) refieren que distintas investigaciones muestran la manifestación de sintomatología ansiosa, depresiva, pánico, angustia, fobia y respuesta estresante a nivel general en la población sobre todo al inicio de la pandemia, sosteniendo que la presencia de ansiedad o ausencia de ella repercute a nivel comportamental en el individuo como, por ejemplos, actos disruptivos o la compulsión en el cuidado exagerando medidas de protección e higiene así como agudizar la presencia de una pequeña dolencia interpretándola como contagio mientras que, por el contrario, la falta de ansiedad puede hacer creer al sujeto que las medidas de prevención o cuidado para prevenir la enfermedad son innecesarios, por tanto, pueden incumplir los protocolos de cuidado actuando de 
forma irresponsable consigo mismo y con los otros. Además, niveles muy elevados de ansiedad conllevan a comportamientos irracionales porque afecta su capacidad de toma de decisiones.

Gonzalez et alt. (2021) citando a Khan et al. (2020) manifiestan que las personas que fueron sometidas a confinamiento por pandemia suelen experimentar ansiedad, pánico, alteraciones del sueño, del humor y desesperanza. El mismo autor citan a Wang et al. (2020) identificaron indicadores de depresión, ansiedad y estrés en niveles moderado a severo en la muestra que fue objeto de su estudio a inicios de la pandemia, asimismo citando a Huang y Zhao (2020) se evidencia la presencia de ansiedad, depresión y alteraciones del sueño también suscitados en la primera fase del confinamiento en China. Del mismo modo, el autor cita a Ozamiz et al. (2020) en una investigación realizada en España muestran que son los jóvenes los más propensos a presentar niveles elevados de estrés, ansiedad y depresión en comparación a personas adultas además de que los pacientes crónicos tienen mayor impacto en su salud mental.

Álvarez et al. (2020), citando a Rodríguez et al. (2020), manifiestan que los niveles de ansiedad en los estudiantes son causados por: el cúmulo de trabajos, la ejecución inadecuada de sus cursos virtuales, el no acompañamiento pedagógico, entre otros. Además, refieren que las reacciones más frecuentes en los estudiantes se vinculan con el temor y la incertidumbre presentándose comportamientos que evidencian angustia, ansiedad, depresión somatización, entre otros. Asimismo, Wang et al. (2020) evidenciaron el incremento de los niveles ansiosos, depresivos e indignación lo que a su vez produjo la disminución de aspectos asociados a felicidad y satisfacción con la vida. De acuerdo con Velásquez (2020) en Sudamérica, existen estudiantes del primer año de la facultad de ciencias de la salud con sintomatología ansiosa, depresiva y estrés en un nivel grave y medio siendo los porcentajes $23 \&, 39 \%$ y $31 \%$ respectivamente.

En el Perú, los índices de ansiedad oscilan de 9,1\% a 46,5\% en los primeros ciclos y años de estudio en carreras no ligadas a ciencias de la salud. Digión y Álvarez (2021) manifiesta que la ansiedad afecta el aprendizaje afectando su capacidad perceptiva lo que se manifiesta con la poca o escasa retención del contenido estudiado aumento el riesgo de fracaso académico lo que conlleva a prácticas nocivas como el consumo de alcohol y tabaco. Lamentablemente, en nuestro país existe un escaso conocimiento respecto a la ansiedad y el estrés de tipo académico donde, si bien es cierto hay estudios exploratorios 
conducentes a grados en universidades distintas a Áncash, existe un escaso rigor metodológico por lo que resulta importante generar estudios que contemplen estas variables.

\subsection{Estrés académico en universitarios}

Estrada et al. (2020) sostienen que la prevalencia del estrés de tipo académico en estudiantes del nivel superior se debe a un conjunto de agentes estresores entre los cuales se muestran: la exigencia en el desarrollo de actividades académicas, el poco tiempo que se asignan a los trabajos, las horas de conexión a clase, la evaluación y actividades en línea. Ascue y Loa (2021) citando a Morales et al. (2016), quienes a su vez fueron citados por Moya, Nieves y Soldado (2020) sostienen que la educación virtual es un medio que permite propiciar el acceso, viabilidad y calidad del nivel educativo en los distintos contextos educativos. En ese sentido, debido a que el rol de la universidad constituye fomentar el logro y desarrollo de competencias en sus estudiantes a través de la formación, investigación y la responsabilidad de índole social, se vieron obligados a implementar el uso de plataformas y medios digitales para continuar con el servicio educativo en el contexto Covid-19 pues, al inicio de la cuarentena, los estudiantes se vieron en la necesidad de permanecer confinados en sus casas conectándose a sus clases por computadora u otro dispositivo móvil generando situaciones poco motivadores en su proceso de enseñanza-aprendizaje.

González (2020) manifiestan que la suspensión de actividades académicas sorprendió a todos los agentes educativos que los persuadieron en optar por la virtualización de sus contenidos y recursos desencadenando estrés y ansiedad ante múltiples factores como: incertidumbre sobre la deserción, temor al contagio, pérdida de empleo o quienes costean los estudios de los propios estudiantes. Barraza (2020) manifiestan que las políticas sanitarias que conllevaron al confinamiento a fin de evitar la proliferación del contagio, así como la suspensión de actividades de tipo social, recreativa o laboral han incrementado los niveles de estrés en la población la cual se agrava por la expectativa de cuándo o cómo volveremos a la tradicional normalidad.

Asimismo, como sostienen Figallo et al. (2020) se desconoce las secuelas de la pandemia que, al retornar, se volverá a la vieja o una nueva normalidad que va a reinventar el sistema o se atenuarán las brechas de desigualdad aún existentes. De acuerdo al informe realizado por el Instituto Internacional para la Educación Superior en América Latina y 
el Caribe de la UNESCO (IESALC, 2020) manifiesta que el impacto de la salud emocional en los estudiantes del nivel superior se presenta con motivo de la restricción de contacto social, así como su rutina cotidiana que afectará el equilibrio emocional principalmente de aquellos estudiantes con problemas psicológicos pre existentes. En ese sentido, Aleman et al. (2020) manifiestan que las secuelas del cambio abrupto que condujo esta pandemia repercute significativamente en el nivel de motivación del estudiante y pone en riesgo su normal desempeño, frente a ello, según estudios realizados en países árabes y asiáticos se han identificado la puesta en marcha de factores preventivos y/o protectores como: religiosidad, familia y sobre todo el acompañamiento pedagógico que reciben los educandos por parte de sus docentes.

\subsection{Acompañamiento pedagógico en pandemia}

Ante el contexto descrito, las universidades consideran que no basta con adiestrar a los docentes en materia de educación virtual, sino que puedan llevar a cabo actos o medidas que le permitan responder a la demanda de la comunidad educativa en esta nueva coyuntura, así como fortalecer su actividad docente. Por ejemplo, un abordaje ecológico y sistémico que promuevan un acercamiento y mayor proximidad entre el docente y el estudiante que hagan posible la innovación y transformación de nuevas experiencias del aprendizaje en el nivel superior. En ese sentido, la formación y el acompañamiento pedagógico universitario articulan la puesta en marcha de aspectos como la reflexión, investigación e innovación que permitan generar propuestas que conduzcan a la diseñar e implementar estrategias que conlleven a contribuir con la mejora de los estudiantes que les asista en su formación universitaria con el objetivo de afrontar este contexto inédito a causa de la pandemia generando una alteración ddel quehacer educativo que requieren medidas urgentes y significativas de acuerdo con Cascante y Villanueva (2020).

Chacón (2021), refiere que en el contexto de licenciamiento institucional en las instituciones de nivel superior por parte de la Superintendencia de Educación Superior (SUNEDU) como uno de los indicadores a evaluar representa la acción tutorial o acompañamiento pedagógico como la alternativa más viable a fin de optimizar el desempeño docente, esta estrategia es implementada en las instituciones educativas a fin de atender la necesidad técnica y de asistencia que requieren los docentes con el objetivo de garantizar el logro de competencias en sus educandos. En ese sentido, la tutoría académica es integral, buscando potenciar habilidades, aprendizajes y competencias en 
esta modalidad no presencial a fin que pueda alcanzar el perfil esperado en su formación profesional. Asimismo, es importante:

a) identificar los componentes de la acción tutorial,

b) identificar las competencias profesionales a desarrollar según el perfil,

c) descubrir las competencias del tutor y

d) explicar las estrategias de la tutoría y acompañamiento pedagógico a implementar en la universidad.

Díaz (2021), citando a De la Cruz (2018), refiere que la tutoría es una forma de acompañamiento que emplean las instituciones educativas para afrontar problemas en el ámbito educativo sea de tipo académico o algún otro. La tutoría, o acompañamiento pedagógico muestra la relación que existe entre el tutorado y el tutor quien representa su guía y mentor. Asimismo, es importante considerar sus elementos: educando, educador e institución siendo esta última la que le provee valor adicional al valor en el nivel superior según refiere el mismo autor citando a Rodríguez (2004). En ese sentido, López (2017) señala que la tutoría es un campo de potencial actuación donde se destaca el apoyo, asesoría, orientación y formación en las dimensiones de tipo personal, social, profesional e incluso académica.

Lobato et al. (2016) sostienen que la tutoría contemporánea se orienta también al acompañamiento en proyectos de índole personal y profesional de los educandos, por tanto, implementar estrategias de acompañamiento pedagógico y/o acción tutorial involucra cambios en la cultura de la universidad, capacitación docente, cambio organizacional en las instituciones y el uso de las tecnologías. Respecto al desarrollo tecnológico, basados en el contexto actual, se puede hablar de e-tutoría y el uso de plataformas que propicien este espacio por la flexibilidad y los canales de comunicación que proveen. En el caso de la Universidad Autónoma de Tamaulipas se hace uso de la plataforma Microsoft Teams para el desarrollo de actividades lectivas y no lectivas en el caso de los docentes incluyendo los programas de acompañamiento y tutoría.

García y Mendía (2015) hablan del acompañante-educador o el educador-acompañante quien, a través de su experiencia y capacidad, contribuye con el desarrollo de quienes constituyen el centro del proceso educativo, los estudiantes. El acompañante pedagógico aporta, con su crítica constructivista y empatía, en el trazo y logro del proyecto de cada estudiante. Por tanto, su acompañamiento se cimienta en una relación cálida, entusiasta, 
motivada por el espíritu compartido de realización del proyecto propuesto por el estudiante, promoviendo la convivencia y comunicación dentro de los marcos del respeto y la autonomía, cediendo al estudiante el protagonismo dentro del proceso educativo a fin que se sientan con la capacidad de poder asumir en rol relevante y transformador.

En los últimos años, la incorporación de recursos digitales, aplicativos, simuladores, páginas web indujo a que los docentes se adapten al uso de estas herramientas y que se han implementado como una nueva competencia basado en la virtualidad. Velásquez (2020) refiere que la tutoría virtual representa un proceso de acompañar al estudiante en su proceso de enseñanza-aprendizaje que combina estrategias para garantizar la formación integral y el desarrollo del educando, donde debido al contexto actual se hizo imperativo el uso de plataformas y herramientas digitales. Sesento (2019) refiere que la tutoría constituye un proceso de acompañamiento que permite recibir a los educandos y que bajo la modalidad virtual permite una conexión síncrona o asíncrona haciendo uso de plataformas basado en el modelo ADIE, es decir, analizar, diseñar, implementar y evaluar. Asimismo, Velásquez (2020) citando a Arbizu (1994) y García (2001) refiere que el rol del tutor virtual en el ámbito universitario es imperativo el desarrollo de otras competencias que la sociedad actualmente demanda, tomando en cuenta que la sociedad es cambiante porque los seres humanos son cambiantes y entre los roles del tutor destacan: promover relaciones humanas, facilitar el aprendizaje, uso de recursos virtuales, orientación y consejería, entre otros.

\section{ESTRATEGIAS METODOLÓGICAS O MATERIALES Y MÉTODOS}

\subsection{Participantes}

Participaron 136 estudiantes de psicología de una universidad privada de Chimbote. La edad promedio fue de 17 a 35 años elegidos mediante muestreo por conveniencia y considerando los criterios de inclusión. Entre los criterios de inclusión se contemplan: Estudiantes matriculados semestre 2020-02, estudiantes de ambos sexos, estudiantes regulares de primera matricula y estudiantes que decidieron participar del presente estudio firmando consentimiento informado.

\subsection{Instrumentos de medida}

Cuestionario de Ansiedad ante los exámenes (CAEX) de Valera, 1999. Para la otra variable se empleó el Inventario SISCO de estrés académico de Barraza, 2002. Para la interpretación de resultados se utilizó la prueba de comparación medias relacionadas a 
partir del estadístico de Wilcoxon, el cual nos proporciona el valor de Z=-5.766 y una probabilidad $\mathrm{p}<0.05$, por cuanto podemos concluir que la aplicación del acompañamiento pedagógico en el sistema educativo no presencial es significativa para disminuir el estrés académico y ansiedad ante los exámenes, cada variable con sus respectivas dimensiones.

\subsection{Procedimiento}

En principio, mediante una carta dirigida al área académica de una universidad privada de Chimbote, se solicitó la autorización correspondiente para poder llevar a cabo la investigación. Dicho documento especificaba el título, los objetivos, el instrumento de evaluación psicológica a emplear, así como los principios éticos contemplados. Luego de recibir la autorización, se procedió con la presentación a los estudiantes a quienes se les pidió firmar el consentimiento informado con el permiso correspondiente de los docentes de turno, los estudiantes fueron previamente informados de la intencionalidad del estudio, así como garantizar la confidencialidad de la información, así como los fines académicos de la misma. En ese sentido, los participantes que cumplieron los criterios de inclusión fueron considerados para esta investigación.

La presente investigación, de acuerdo con Hernández et al. (2018) es pre experimental y según el modelo pragmático es de tipo cuantitativa. El diseño, basado en Gallardo (2017) es pre experimental de un solo grupo. Para la presente investigación se realizó una pre y post prueba, la primera de forma diagnóstica y la segunda para determinar el efecto del programa de acompañamiento pedagógico. Para el recojo de información se emplearon los siguientes instrumentos:

\subsection{Análisis de datos}

Dado que las muestras son superiores al 50 se realizó la prueba de normalidad a partir del estadístico Kolmogorv-smirov, asimismo podemos apreciar que los p-valores son superiores al 5\% demostrando que cada variable se distribuye de manera normal y que la prueba de comparación a realizar en los valores encontrados antes y después de la aplicación del programa correspondería a la T- Student. Los análisis estadísticos se llevaron a cabo en el software estadístico SPSS versión 26 y los datos fueron previamente tabulados en Excell. 


\section{RESULTADOS Y DISCUSIÓN}

\section{Tabla 1}

Niveles de estrés académico

\begin{tabular}{|c|c|c|c|c|c|}
\hline & \multicolumn{4}{|c|}{ Grupo } \\
\hline & & \multicolumn{2}{|c|}{ Pre } & \multicolumn{2}{|c|}{ Post } \\
\hline & & $\mathbf{N}$ & $\%$ & $\mathbf{N}$ & $\%$ \\
\hline \multirow{5}{*}{ Agentes estresores } & Muy bajo & 6 & $4.4 \%$ & 22 & $16.2 \%$ \\
\hline & Bajo & 24 & $17.6 \%$ & 52 & $38.2 \%$ \\
\hline & Promedio & 51 & $37.5 \%$ & 37 & $27.2 \%$ \\
\hline & Alto & 47 & $34.6 \%$ & 20 & $14.7 \%$ \\
\hline & Muy alto & 8 & $5.9 \%$ & 5 & $3.7 \%$ \\
\hline \multirow{5}{*}{ Reacciones Físicas } & Muy bajo & 11 & $8.1 \%$ & 34 & $25.0 \%$ \\
\hline & Bajo & 52 & $38.2 \%$ & 60 & $44.1 \%$ \\
\hline & Promedio & 26 & $19.1 \%$ & 20 & $14.7 \%$ \\
\hline & Alto & 31 & $22.8 \%$ & 16 & $11.8 \%$ \\
\hline & Muy alto & 16 & $11.8 \%$ & 6 & $4.4 \%$ \\
\hline \multirow{5}{*}{$\begin{array}{l}\text { Reacciones } \\
\text { Psicológicas }\end{array}$} & Muy bajo & 3 & $2.2 \%$ & 30 & $22.1 \%$ \\
\hline & Bajo & 36 & $26.5 \%$ & 41 & $30.1 \%$ \\
\hline & Promedio & 48 & $35.3 \%$ & 49 & $36.0 \%$ \\
\hline & Alto & 41 & $30.1 \%$ & 14 & $10.3 \%$ \\
\hline & Muy alto & 8 & $5.9 \%$ & 2 & $1.5 \%$ \\
\hline \multirow{5}{*}{$\begin{array}{l}\text { Reacciones } \\
\text { Comportamentales }\end{array}$} & Muy bajo & 7 & $5.1 \%$ & 38 & $27.9 \%$ \\
\hline & Bajo & 27 & $19.9 \%$ & 46 & $33.8 \%$ \\
\hline & Promedio & 27 & $19.9 \%$ & 24 & $17.6 \%$ \\
\hline & Alto & 45 & $33.1 \%$ & 20 & $14.7 \%$ \\
\hline & Muy alto & 30 & $22.1 \%$ & 8 & $5.9 \%$ \\
\hline \multirow{5}{*}{$\begin{array}{l}\text { Estrategias } \\
\text { afrontamiento }\end{array}$} & Muy bajo & 18 & $13.2 \%$ & 38 & $27.9 \%$ \\
\hline & Bajo & 33 & $24.3 \%$ & 55 & $40.4 \%$ \\
\hline & Promedio & 25 & $18.4 \%$ & 15 & $11.0 \%$ \\
\hline & Alto & 46 & $33.8 \%$ & 20 & $14.7 \%$ \\
\hline & Muy alto & 14 & $10.3 \%$ & 8 & $5.9 \%$ \\
\hline \multirow{5}{*}{ Estrés } & Muy bajo & 0 & $0.0 \%$ & 7 & $5.1 \%$ \\
\hline & Bajo & 16 & $11.8 \%$ & 77 & $56.6 \%$ \\
\hline & Promedio & 79 & $58.1 \%$ & 44 & $32.4 \%$ \\
\hline & Alto & 40 & $29.4 \%$ & 7 & $5.1 \%$ \\
\hline & Muy alto & 1 & $0.7 \%$ & 1 & $0.7 \%$ \\
\hline Total & & 136 & $100.0 \%$ & 136 & $100.0 \%$ \\
\hline
\end{tabular}

Nota: Estrés académico antes y después de aplicar el acompañamiento pedagógico en el sistema de educación no presencial, bajo el contexto del COVID-19, en estudiantes de una universidad privada, Chimbote-2020 


\section{.Tabla 2}

Niveles de ansiedad frente a los exámenes

\begin{tabular}{|c|c|c|c|c|c|}
\hline & \multicolumn{4}{|c|}{ Grupo } \\
\hline & & \multicolumn{2}{|c|}{ Pre } & \multicolumn{2}{|c|}{ Post } \\
\hline & & $\mathbf{N}$ & $\%$ & $\mathbf{N}$ & $\%$ \\
\hline \multirow{4}{*}{$\begin{array}{l}\text { Reacciones } \\
\text { fisiológicas }\end{array}$} & Muy bajo & 2 & $1.5 \%$ & 58 & $42.6 \%$ \\
\hline & Bajo & 55 & $40.4 \%$ & 51 & $37.5 \%$ \\
\hline & Promedio & 68 & $50.0 \%$ & 19 & $14.0 \%$ \\
\hline & Alto & 11 & $8.1 \%$ & 8 & $5.9 \%$ \\
\hline \multirow{4}{*}{$\begin{array}{l}\text { Respuestas de } \\
\text { evitación }\end{array}$} & Muy bajo & 0 & $0.0 \%$ & 55 & $40.4 \%$ \\
\hline & Bajo & 21 & $15.4 \%$ & 44 & $32.4 \%$ \\
\hline & Promedio & 91 & $66.9 \%$ & 29 & $21.3 \%$ \\
\hline & Alto & 24 & $17.6 \%$ & 8 & $5.9 \%$ \\
\hline \multirow{4}{*}{$\begin{array}{l}\text { Situaciones } \\
\text { ansiosas }\end{array}$} & Muy bajo & 5 & $3.7 \%$ & 29 & $21.3 \%$ \\
\hline & Bajo & 53 & $39.0 \%$ & 62 & $45.6 \%$ \\
\hline & Promedio & 77 & $56.6 \%$ & 39 & $28.7 \%$ \\
\hline & Alto & 1 & $0.7 \%$ & 6 & $4.4 \%$ \\
\hline \multirow{4}{*}{ Preocupación } & Muy bajo & 13 & $9.6 \%$ & 41 & $30.1 \%$ \\
\hline & Bajo & 54 & $39.7 \%$ & 46 & $33.8 \%$ \\
\hline & Promedio & 68 & $50.0 \%$ & 47 & $34.6 \%$ \\
\hline & Alto & 1 & $0.7 \%$ & 2 & $1.5 \%$ \\
\hline \multirow{4}{*}{ Ansiedad } & Ausencia de ansiedad & 0 & $0.0 \%$ & 27 & $19.9 \%$ \\
\hline & Ansiedad leve & 51 & $37.5 \%$ & 75 & $55.1 \%$ \\
\hline & Ansiedad moderada & 81 & $59.6 \%$ & 29 & $21.3 \%$ \\
\hline & $\begin{array}{l}\text { Grave presencia de } \\
\text { ansiedad }\end{array}$ & 4 & $2.9 \%$ & 5 & $3.7 \%$ \\
\hline Total & & 136 & $100.0 \%$ & 136 & $100.0 \%$ \\
\hline
\end{tabular}

Nota: Ansiedad frente a los exámenes antes y después de aplicar el acompañamiento pedagógico en el sistema de educación no presencial, bajo el contexto del COVID-19, en estudiantes de una universidad privada, Chimbote-2020 
Tabla 3.

Dimensiones del estrés académico

\begin{tabular}{|c|c|c|c|}
\hline & \multicolumn{2}{|c|}{ Grupo } \\
\hline & & Pre & Post \\
\hline \multirow{5}{*}{ Agentes estresores } & Media & 25.8 & 21.3 \\
\hline & Escala & (Promedio) & (Promedio) \\
\hline & Desviación estándar & 6.0 & 6.3 \\
\hline & Varianza & 35.8 & 39.4 \\
\hline & Coeficiente de Variación & $23.2 \%$ & $29.5 \%$ \\
\hline \multirow{5}{*}{ Reacciones Físicas } & Media & 17.8 & 14.3 \\
\hline & Escala & (Promedio) & (Promedio) \\
\hline & Desviación estándar & 5.6 & 5.6 \\
\hline & Varianza & 31.6 & 31.8 \\
\hline & Coeficiente de Variación & $31.6 \%$ & $39.4 \%$ \\
\hline \multirow{5}{*}{$\begin{array}{l}\text { Reacciones } \\
\text { Psicológicas }\end{array}$} & Media & 14.9 & 12.2 \\
\hline & Escala & (Promedio) & (Bajo) \\
\hline & Desviación estándar & 3.8 & 4.0 \\
\hline & Varianza & 14.7 & 15.9 \\
\hline & Coeficiente de Variación & $25.7 \%$ & $32.6 \%$ \\
\hline \multirow{5}{*}{$\begin{array}{l}\text { Reacciones } \\
\text { Comportamentales }\end{array}$} & Media & 13.5 & 9.9 \\
\hline & Escala & (Promedio) & (Bajo) \\
\hline & Desviación estándar & 3.7 & 4.1 \\
\hline & Varianza & 14.0 & 16.4 \\
\hline & Coeficiente de Variación & $27.7 \%$ & $40.9 \%$ \\
\hline \multirow{5}{*}{$\begin{array}{l}\text { Estrategias } \\
\text { afrontamiento }\end{array}$} & Media & 17.9 & 14.5 \\
\hline & Escala & (Promedio) & (Bajo) \\
\hline & Desviación estándar & 6.1 & 6.1 \\
\hline & Varianza & 37.3 & 37.3 \\
\hline & Coeficiente de Variación & $34.0 \%$ & $42.2 \%$ \\
\hline \multirow{5}{*}{ Estrés } & Media & 89.9 & 72.2 \\
\hline & Escala & (Promedio) & (Bajo) \\
\hline & Desviación estándar & 13.5 & 14.2 \\
\hline & Varianza & 183.0 & 201.6 \\
\hline & Coeficiente de Variación & $15.0 \%$ & $19.7 \%$ \\
\hline
\end{tabular}

Nota: Dimensiones del estrés académico antes y después de aplicar el acompañamiento pedagógico en el sistema de educación no presencial, bajo el contexto del COVID-19, en estudiantes de una universidad privada, Chimbote-2020. 


\section{Tabla 4}

Dimensiones de la ansiedad frente a los exámenes

\begin{tabular}{|c|c|c|c|}
\hline & & \multicolumn{2}{|c|}{ Grupo } \\
\hline & & Pre & Post \\
\hline \multirow{5}{*}{$\begin{array}{l}\text { Reacciones } \\
\text { fisiológicas }\end{array}$} & Media & 48.1 & 36.4 \\
\hline & Escala & (Promedio) & (Bajo) \\
\hline & Desviación estándar & 8.7 & 12.2 \\
\hline & Varianza & 76.1 & 149.8 \\
\hline & Coeficiente de variación & $18.1 \%$ & $33.6 \%$ \\
\hline \multirow{5}{*}{$\begin{array}{l}\text { Respuestas de } \\
\text { evitación }\end{array}$} & Media & 25.1 & 18.2 \\
\hline & Escala & (Promedio) & (Bajo) \\
\hline & Desviación estándar & 3.6 & 6.9 \\
\hline & Varianza & 13.2 & 47.0 \\
\hline & Coeficiente de variación & $14.5 \%$ & $37.6 \%$ \\
\hline \multirow{5}{*}{$\begin{array}{l}\text { Situaciones } \\
\text { ansiosas }\end{array}$} & Media & 44.2 & 39.4 \\
\hline & Escala & (Promedio) & (Bajo) \\
\hline & Desviación estándar & 9.9 & 11.8 \\
\hline & Varianza & 97.5 & 138.4 \\
\hline & Coeficiente de variación & $22.4 \%$ & $29.8 \%$ \\
\hline \multirow{5}{*}{ Preocupación } & Media & 43.1 & 37.2 \\
\hline & Escala & (Promedio) & (Bajo) \\
\hline & Desviación estándar & 11.0 & 13.1 \\
\hline & Varianza & 120.9 & 170.5 \\
\hline & Coeficiente de variación & $25.5 \%$ & $35.1 \%$ \\
\hline \multirow{5}{*}{ Ansiedad } & Media & 160.4 & 131.2 \\
\hline & Escala & (Promedio) & (Bajo) \\
\hline & Desviación estándar & 20.7 & 33.6 \\
\hline & Varianza & 427.8 & 1129.7 \\
\hline & Coeficiente de variación & $12.9 \%$ & $25.6 \%$ \\
\hline
\end{tabular}

Nota: Dimensiones de la ansiedad frente a los exámenes antes y después de aplicar el acompañamiento pedagógico en el sistema de educación no presencial, bajo el contexto del COVID-19, en estudiantes de una universidad privada, Chimbote-2020. 


\section{Tabla 5}

Prueba de normalidad del estrés académico y ansiedad antes los exámenes

\begin{tabular}{|c|c|c|c|c|}
\hline \multirow{2}{*}{ Grupo } & & \multicolumn{3}{|c|}{ Kolmogorov-Smirnov ${ }^{\mathbf{a}}$} \\
\hline & & Estadístico & gl & Sig. \\
\hline \multirow{2}{*}{ Agentes estresores } & Pre & 0.084 & 136 & 0.019 \\
\hline & Post & 0.096 & 136 & 0.004 \\
\hline \multirow{2}{*}{ Reacciones Físicas } & Pre & 0.153 & 136 & 0.000 \\
\hline & Post & 0.158 & 136 & 0.000 \\
\hline \multirow{2}{*}{ Reacciones Psicológicas } & Pre & 0.101 & 136 & 0.002 \\
\hline & Post & 0.138 & 136 & 0.000 \\
\hline \multirow{2}{*}{ Reacciones Comportamentales } & Pre & 0.109 & 136 & 0.000 \\
\hline & Post & 0.159 & 136 & 0.000 \\
\hline \multirow{2}{*}{ Estrategias de afrontamiento } & Pre & 0.133 & 136 & 0.000 \\
\hline & Post & 0.194 & 136 & 0.000 \\
\hline \multirow{2}{*}{ Estrés } & Pre & 0.067 & 136 & ,200* \\
\hline & Post & 0.093 & 136 & 0.006 \\
\hline \multirow{2}{*}{ Reacciones fisiológicas } & Pre & 0.073 & 136 & 0.075 \\
\hline & Post & 0.126 & 136 & 0.000 \\
\hline \multirow{2}{*}{ Respuestas de evitación } & Pre & 0.073 & 136 & 0.069 \\
\hline & Post & 0.136 & 136 & 0.000 \\
\hline \multirow{2}{*}{ Situaciones ansiosas } & Pre & 0.060 & 136 & ,200* \\
\hline & Post & 0.083 & 136 & 0.024 \\
\hline \multirow{2}{*}{ Preocupación } & Pre & 0.057 & 136 & ,200* \\
\hline & Post & 0.074 & 136 & 0.064 \\
\hline \multirow{2}{*}{ Ansiedad } & Pre & 0.073 & 136 & 0.072 \\
\hline & Post & 0.057 & 136 & ,200* \\
\hline
\end{tabular}

Nota: La tabla presenta los resultados de la prueba de bondad de ajuste de KolmogorovSmirnov, la para grupos compuestos por más de 50 sujetos. Se observa que la variable ansiedad, el estrés y sus respectivas dimensiones, tanto en el pre test como en el post test, presentaron distribución no normal $(\mathrm{p}<0,05)$; en tal sentido, todas las hipótesis de comparación entre el grupo pre y post, deberán contrastarse con una estadística no paramétrica: Wilcoxon para grupos relacionados. 


\section{Tabla 7}

Prueba de comparación de medias para los niveles de estrés académico

Estadísticos de prueba ${ }^{a}$

\begin{tabular}{lccccc}
\hline & $\begin{array}{c}\text { Agentes } \\
\text { estresores } \\
\text { Pre-Post }\end{array}$ & $\begin{array}{c}\text { Reacciones } \\
\text { Físicas } \\
\text { Pre-Post }\end{array}$ & $\begin{array}{c}\text { Reacciones } \\
\text { Psicológicas } \\
\text { Pre-Post }\end{array}$ & $\begin{array}{c}\text { Estrategias de } \\
\text { afrontamiento } \\
\text { Pre-Post }\end{array}$ & $\begin{array}{c}\text { Estrés } \\
\text { Pre- } \\
\text { Post }\end{array}$ \\
\hline $\mathrm{Z}$ & $-5,766^{\mathrm{b}}$ & $-4,919^{\mathrm{b}}$ & $-5,493^{\mathrm{b}}$ & $-5,125^{\mathrm{b}}$ & - \\
Sig. & & & & & $9,023^{\mathrm{b}}$ \\
asintótica(bilateral) & 0.000 & 0.000 & 0.000 & 0.000 & 0.000 \\
\hline
\end{tabular}

a. Prueba de rangos con signo de Wilcoxon

b. Se basa en rangos negativos.

Nota: La tabla 7 muestra la prueba de comparación medias relacionadas a partir del estadístico de Wilcoxon, el cual nos proporciona el valor de $\mathrm{Z}=-5.766$ y una probabilidad $\mathrm{p}<0.05$, por cuanto se concluye que la aplicación del acompañamiento pedagógico en el sistema educativo no presencial es significativo para disminuir el estrés. Así mismo presenta las pruebas para las dimensiones:

Agentes estresores con un valor de $Z=-5.766$ y $p<0.05$ por tanto se concluye que el acompañamiento pedagógico disminuye significativamente el estrés en la dimensión agentes estresores.

Reacciones físicas con un valor de $Z=-4.919$ y $\mathrm{p}<0.05$ por tanto se concluye que el acompañamiento pedagógico disminuye significativamente el estrés en la dimensión Reacciones físicas

Reacciones psicológicas con valor $\mathrm{Z}=-5.493$ y $\mathrm{p}<0.05$ por tanto se concluye que el acompañamiento pedagógico disminuye significativamente el estrés en la dimensión Reacciones psicológicas.

Estrategias de afrontamiento con valor $Z=-5.125$ para lo cual el valor de significancia $\mathrm{p}=0.000$ es inferior al 5\%. Por tanto se concluye que el acompañamiento pedagógico disminuye significativamente el estrés en la dimensión estrategias de afrontamiento. 


\section{Tabla 8}

Prueba de comparación de medias para los niveles de ansiedad frente a los exámenes

Estadísticos de prueba ${ }^{a}$

\begin{tabular}{lccccc}
\hline & $\begin{array}{c}\text { Reacciones } \\
\text { fisiológicas } \\
\text { Pre - Post }\end{array}$ & $\begin{array}{c}\text { Respuestas } \\
\text { de } \\
\text { evitación } \\
\text { Pre - Post }\end{array}$ & $\begin{array}{c}\text { Situaciones } \\
\text { ansiosas } \\
\text { Pre - Post }\end{array}$ & $\begin{array}{c}\text { Preocupación } \\
\text { Pre - Post }\end{array}$ & $\begin{array}{c}\text { Ansiedad } \\
\text { Pre - } \\
\text { Post }\end{array}$ \\
\hline $\mathrm{Z}$ & $-7,549^{\mathrm{b}}$ & $-8,274^{\mathrm{b}}$ & $-3,816^{\mathrm{b}}$ & $-4,760^{\mathrm{b}}$ & $-8,080^{\mathrm{b}}$ \\
Sig. & 0.000 & 0.000 & 0.000 & 0.000 & 0.000 \\
asintótica(bilateral) & & & & & \\
\hline
\end{tabular}

a. Prueba de rangos con signo de Wilcoxon

b. Se basa en rangos negativos.

Nota: La tabla 8 muestra la prueba de comparación medias relacionadas a partir del estadístico de Wilcoxon, el cual proporciona el valor de $\mathrm{Z}=-8.080$ y una probabilidad $\mathrm{p}<0.05$, por cuanto se concluye que la aplicación del acompañamiento pedagógico en el sistema educativo no presencial es significativo para disminuir la ansiedad frente los exámenes. Así mismo presenta las pruebas para las dimensiones:

Reacciones fisiológicas con un valor de $\mathrm{Z}=-7.549 \mathrm{y} \mathrm{p}<0.05$ por tanto se concluye que el acompañamiento pedagógico disminuye significativamente el estrés en la dimensión Reacciones fisiológicas

Respuestas de evitación con un valor de $\mathrm{Z}=-8.274$ y $\mathrm{p}<0.05$ por tanto se concluye que el acompañamiento pedagógico disminuye significativamente el estrés en la dimensión Respuestas de evitación

Situaciones ansiosas con valor $\mathrm{Z}=-3.816$ y $\mathrm{p}<0.05$ por tanto se concluye que el acompañamiento pedagógico disminuye significativamente el estrés en la dimensión Situaciones ansiosas.

Preocupación con valor $\mathrm{Z}=-4.760$ para lo cual el valor de significancia $\mathrm{p}=0.000$ es inferior al 5\%. Por tanto, se concluye que el acompañamiento pedagógico disminuye significativamente la ansiedad frente a los exámenes en la dimensión Preocupación.

La presente investigación se llevó a cabo con el objetivo de determinar la efectividad del acompañamiento pedagógico para mitigar el estrés académico y la ansiedad ante los exámenes. De acuerdo al análisis de resultados obtenidos en el presente estudio, se identifica que los niveles de las variables mencionadas han disminuido significativamente 
en el post test. Esto quiere decir que el acompañamiento pedagógico realizado a los estudiantes ha tenido un impacto positivo, lo que coincide con los hallazgos de Ménard y Boucher (2018) concluyen que la evolución provocada por la capacitación o acompañamiento es duradera en el tiempo.

Asimismo, antes de aplicar el acompañamiento académico en el sistema educativo no presencial los estudiantes reportaban en un $11.8 \%$ niveles de estrés bajo, el $58.1 \%$ promedio, el $29.4 \%$ alto y el $0.7 \%$ muy alto; después de aplicar el acompañamiento se observa que el $5.1 \%$ presenta niveles muy bajos, el $56.6 \%$ bajos, el $32.4 \%$ promedio, el $5.1 \%$ altos y el $0.7 \%$ muy alto. Además según la dimensión Agentes estresores, se observa que antes de aplicar el acompañamiento académico el $4.4 \%$ presenta niveles muy bajos, el $17.6 \%$ bajo, el $35.7 \%$ promedio, el $34.6 \%$ alto y el $5.9 \%$ muy alto; luego de aplicar el acompañamiento se observó que el $16.2 \%$ presenta un nivel muy bajo, 38.2\% bajo, el $27.2 \%$ promedio, el $14.7 \%$ alto y el $3.7 \%$ muy alto; según la dimensión Reacciones físicas, se observa que antes de aplicar el acompañamiento académico el 8.1\% presenta niveles muy bajos, el $38.2 \%$ bajo, el $19.1 \%$ promedio, el $22.8 \%$ alto y el $11.8 \%$ muy alto; luego de aplicar el acompañamiento se observó que el 25.0\% presenta un nivel muy bajo, $44.1 \%$ bajo, el $14.7 \%$ promedio, el $11.8 \%$ alto y el $4.4 \%$ muy alto; según la dimensión Reacciones psicológicas, se observa que antes de aplicar el acompañamiento académico el $2.2 \%$ presenta niveles muy bajos, el $26.5 \%$ bajo, el $35.3 \%$ promedio, el $30.1 \%$ alto y el 5.9\% muy alto; luego de aplicar el acompañamiento se observó que el $22.1 \%$ presenta un nivel muy bajo, $30.1 \%$ bajo, el $36.0 \%$ promedio, el $10.3 \%$ alto y el $1.5 \%$ muy alto; según la dimensión Reacciones comportamentales, se observa que antes de aplicar el acompañamiento académico el $5.1 \%$ presenta niveles muy bajos, el $19.9 \%$ bajo, el $19.9 \%$ promedio, el $33.1 \%$ alto y el $22.1 \%$ muy alto; luego de aplicar el acompañamiento se observó que el $27.9 \%$ presenta un nivel muy bajo, 33.8\% bajo, el $17.2 \%$ promedio, el $14.7 \%$ alto y el 5.9\% muy alto; finalmente en la dimensión Estrategias de afrontamiento, se observa que antes de aplicar el acompañamiento académico el 13.2\% presenta niveles muy bajos, el $24.3 \%$ bajo, el $18.4 \%$ promedio, el $33.8 \%$ alto y el $10.3 \%$ muy alto; luego de aplicar el acompañamiento se observó que el $27.9 \%$ presenta un nivel muy bajo, $40.4 \%$ bajo, el $11.0 \%$ promedio, el $14.7 \%$ alto y el $5.9 \%$ muy alto.

Por otro lado, antes de aplicar el acompañamiento académico en el sistema educativo no presencial los estudiantes reportaban en un $37.58 \%$ una leve ansiedad ante los exámenes, 
el 59.6\% una ansiedad moderada y el 2.9\% una ansiedad grave, luego de aplicar el acompañamiento, el $19.9 \%$ no presento ansiedad, el 55.1\% una ansiedad leve, el $21.3 \%$ una ansiedad moderada y el 3.7\% ansiedad grave. En la dimensión Reacciones fisiológica se observa que, antes de aplicar el acompañamiento académico el $1.5 \%$ presenta una ansiedad muy baja, el $40.4 \%$ baja, el $50.0 \%$ promedio y el $8.1 \%$ alto; luego de aplicar el acompañamiento se tiene que el $42.6 \%$ presenta una ansiedad muy baja, el $37.5 \%$ baja, el 14.0\% promedio y el 5.9\% alto; En la dimensión Respuesta de evitación se evidencia que, antes de aplicar el acompañamiento académico el 15.4\% presenta una ansiedad baja, el $66.9 \%$ promedio y el $17.6 \%$ alto; luego de aplicar el acompañamiento se tiene que el $40.4 \%$ presenta una ansiedad muy baja, el $32.4 \%$ baja, el $21.3 \%$ promedio y el $5.9 \%$ alto; en la dimensión Situaciones ansiosas se observa que, antes de aplicar el acompañamiento académico el $3.7 \%$ presenta una ansiedad muy baja, el $39.0 \%$ baja, el $56.6 \%$ promedio y el $0.7 \%$ alto; luego de aplicar el acompañamiento se tiene que el $21.3 \%$ presenta una ansiedad muy baja, el $45.6 \%$ baja, el $28.7 \%$ promedio y el $4.4 \%$ alto; finalmente la dimensión Preocupación se muestra que, antes de aplicar el acompañamiento académico el $9.6 \%$ presenta una ansiedad muy baja, el $39.7 \%$ baja, el $50.0 \%$ promedio y el $0.7 \%$ alto; luego de aplicar el acompañamiento se tiene que el $30.1 \%$ presenta una ansiedad muy baja, el $33.8 \%$ baja, el $34.6 \%$ promedio y el $1.5 \%$ alto.

\section{CONCLUSIÓN O CONSIDERACIONES FINALES}

Se concluye que el contexto COVID-19 trajo consigo una repercusión psicológica y social no prevista pues significó un cambio de rumbo en el sistema de educación tradicional a nivel superior, tal como sostienen Alemán et al. (2020) en todo proceso social se producen situaciones adversas como ésta que deben verse como oportunidad de respuesta adaptativa que permitan implementar nuevas propuestas pedagógicas que permitan responder positivamente en la formación de futuros profesionales. Por tanto, es conveniente saber aprovechar los medios y recursos que permitan adaptarse al nuevo contexto pues los efectos de esta pandemia significaron un giro en la educación del nivel superior. Coincidiendo con García et al. (2021) quienes sostienen que toda población, incluyendo la universitaria, están expuestas a contraer enfermedades que repercuten en su salud emocional por lo que resulta urgente el diseño e implementación de estrategias que permitan su temprana detección pues la actual pandemia ha denotado la necesidad de mejorar o proponer programas de intervención en salud mental a nivel superior, así como 
validar instrumentos de evaluación remota. En ese sentido, un programa de acompañamiento pedagógico y/o psicopedagógico resulta significativo para poder brindar a los estudiantes los medios y herramientas necesarias que les permita mitigar el impacto del actual contexto, como reducir sus niveles de estrés académico y ansiedad ante los exámenes, pudiendo emplear dichas estrategias a lo largo de su formación universitaria.

\section{REFERENCIAS BIBLIOGRAFICAS}

Acevedo, K. y Amador, D. (2021). Estrés, estrategias de afrontamiento y experiencia académica en estudiantes universitarios en tiempos de pandemia delCOVID-19. La experiencia de Nicaragua y Chile. Revista Torreón Universitario, 10(27), 1-4. https://doi.org/10.5377/torreon.v10i27.10839

Alania, R., Llancari, R., Rafaele de la Cruz, M., y Ortega, D. (2020). Adaptación del Inventario de Estrés Académico SISCO SV al contexto de la crisis por COVID19. Socialium, 4(2), 111-130. https://doi.org/10.26490/uncp.sl.2020.4.2.669

Área, M., Bethencourt, A., Gómez, M. (2020). De la enseñanza semipresencial a la enseñanza online en tiempos de Covid19. Revista Campus Virtuales, 9(2), 35-50. http://www.uajournals.com/campusvirtuales/images/numeros/17.pdf

Ascue, R. y Loa, R. (2020). Estrés académico en los estudiantes de las universidades públicas del a Región. Apurímac en tiempos de pandemia Covid-19. Ciencia Latina Revista Científica Multidisciplinar. 5(1). 367-369. https://doi.org/10.37811/cl_rcm.v5i1.238

Barraza, A. (2019). Estrés académico en alumnos de tres niveles educativos, un estudio comparativo. Praxis Investigativa REDIE, 11(21), 149-163. https://dialnet.unirioja.es/servlet/articulo? codigo=7145135

Cabero, J. y Llorente, C. (2020). Covid-19: transformación radical de la digitalización en las instituciones. Revista Campus Virtuales, 9(2), 25-34. http://www.uajournals.com/campusvirtuales/images/numeros/17.pdf

Castro, M., Paz, M. y Cela, E. (2020). Aprendiendo a enseñar en tiempos de pandemia COVID-19: nuestra experiencia en una universidad pública de Argentina. Rev. Digit. Invest. Docencia Univ. 14(2), 2-3. https://doi.org/10.19083/ridu.2020.1271 
Chacon, M. (2021). Acción tutorial en el fortalecimiento del perfil profesional universitario: aportes en el desarrollo de competencias a partir de la educación virtual. DOI: 10.48082/espacios-a21v42n05p05

Comisión Económica para América Latina y el Caribe (CEPAL,2020) La educación en tiempos de la pandemia de COVID-19. (1), 3-5. https://repositorio.cepal.org/bitstream/handle/11362/45904/1/S2000510_es.pdf

Diaz, J. (2021). Administración de la práctica tutorial en tiempos del COVID-19: Atendiendo las necesidades especiales de los alumnos universitarios a través de la tutoría en línea. https://doi.org/10.46377/dilemas.v8i.2557

Digión, L. y Álvarez, M. (2021). Experiencia de enseñanza - aprendizaje con aula virtual en el acompañamiento pedagógico debido al Covid-19, 13(1), 20-34. http://doi.org/10.32870/Ap.v13n1.1957

Estrada, E., Mamani, M., Gallegos, N., Mamani, H., Zuloaga, M. (2021) Estrés académico en estudiantes universitarios peruanos en tiempos de la pandemia del COVID-19. Revista AVFT, 40(1). 88-89. http://doi.org/10.5281/zenodo.4675923

Figallo, F., Gonzalez, M., Diestra, V. (2020) Perú: educación superior en el contexto de la pandemia por el covid-19. Revista de Educación Superior en América Latina.

http://rcientificas.uninorte.edu.co/index.php/esal/article/viewFile/13404/214421 444832

Gallardo, E. (2017). Metodología de la investigación ( $1^{\mathrm{a}}$ edición). Universidad Continental. https://repositorio.continental.edu.pe/bitstream/20.500.12394/4278/1/DO_UC_E G_MAI_UC0584_2018.pdf

García, P., Ortiz, X., Botello, E., Aguayo, R., Leija, J., Góngora, F. y Gecen Investigators (2021) Psychosocial impact on health-related and non-health related university students during the COVID-19 pandemic. Results of an electronic survey. Revista Colombiana de Psiquiatría, 50(3):214-224. https://doi.org/10.1016/j.rcp.2021.04.008 
Gonzales, L. (2020) Estrés académico en estudiantes universitarios asociados a la pandemia por COVID-19. Espacio i+d, innovación más desarrollo, 9(25), 161166. https://doi.org/10.31644/IMASD.25.2020.a10

Hernández, R. y Mendoza, C. (2018). Metodología de la investigación: las rutas cuantitativa, cualitativa y mixta. México: Mc Graw Hill. http://www.biblioteca.cij.gob.mx/Archivos/Materiales_de_consulta/Drogas_de_ Abuso/Articulos/SampieriLasRutas.pdf

Hernández, A., Ramos, M., Placencia, B., Indocochea, B., Quimis, A. y Moreno, L. (2018). Metodología de la Investigación Científica. Editorial área de innovación y desarrollo. https://corladancash.com/wpcontent/uploads/2020/01/Metodologia-de-la-inv-cientifica-Arturo-AndresHernandez-Escobar.pdf

Lobato, C., Nagore, F., \& Bilbao, G. (2016). La tutoría en la educación superior en Iberoamérica: Avances y desafíos. Educar, 52(2), 379-398. http://dx.doi.org/10.5565/rev/educar.726

López Gómez, E. (2017). El concepto y las finalidades de la tutoría universitaria : una consulta a expertos. Revista Española de Orientación y Psicopedagogía

Ménard, L., Kozanitis, A., Boucher, S. (2018) Capacitación y acompañamiento pedagógico de profesores universitarios noveles: efectos sobre el uso de estrategias de enseñanza. Práxis Educativa, Ponta Grossa, 13(2), 294-311. https://dialnet.unirioja.es/servlet/articulo?codigo $=6844864$

Miguel, J. (2020) La educación superior en tiempos de pandemia: una visión desde dentro del proceso formativo. Revista Latinoamericana de Estudios Educativos. L (e). 13-40. https://www.redalyc.org/jatsRepo/270/27063237017/html/index.html

Nuñez, LL. (2021) Ansiedad y estresores académicos en estudiantes de enfermería. Revista Recien,10(2), 46-47. https://revista.cep.org.pe/index.php/RECIEN/article/view/73/85

Restrepo, J., Sánchez, O. y Castañeda Quirama, T. (2020). Estrés académico en estudiantes universitarios. Revista Psicoespacios, (24), 17-37, DOI: 10.25057/21452776.1331 17 .

Sánchez, H., Yarlequé, L., Leda, E., Nuñez, R., Arenas, C. Matalinares, M., Gutiérrez, E., Egoavil, I., Solis, J. y Fernández, C. (2021). Indicadores de ansiedad, 
depresión, somatización y evitación experiencial en estudiantes universitarios del Perú en cuarentena por covid-19. Rev. Fac. Med. Hum. 21(2):346-353. DOI 10.25176/RFMH.v21i2.3654

Saravia, M., Cazorla, P., Cedillo, L. (2020). Nivel de ansiedad de estudiantes de medicina de primer año de una universidad privada del Perú en tiempos de covid-19. Rev. Fac. Med. Hum. 20(4):568-573. DOI 10.25176/RFMH.v20i4.3198

Velásquez, B. (2020). La educación virtual en tiempos de Covid-19. Universidad de San Carlos de Guatemala 3(1) 19-25. DOI: https://doi.org/10.46734/revcientifica.v2i1.8

Velazquez, L., Valenzuela, C. y Murillo, J. (2020) Pandemia COVID-19: repercusiones en la educación universitaria. Revista Odontología Sanmarquina de la Facultad de Odontología, Universidad Nacional Mayor de San Marcos. 23(2): 203-206. https://docs.bvsalud.org/biblioref/2020/06/1097504/17766-texto-del-articulo62036-1-10-20200507.pdf

Vivanco, A., Saroli, D., Caycho, T., Carbajal, C. y Grijalva, N. (2020). Ansiedad por Covid-19 y salud mental en estudiantes universitarios. Revista de Investigación en Psicología, 23(2), 197-215. http://dx.doi.org/10.15381/rinvp.v23i2.19241 